\title{
2000-2003 Real Estate Bubble in the UK but not in the USA
}

\author{
Wei-Xing Zhou ${ }^{\mathrm{a}}$, Didier Sornette ${ }^{\mathrm{a}, \mathrm{b}, \mathrm{c}, 1}$ \\ ${ }^{a}$ Institute of Geophysics and Planetary Physics, University of California Los \\ Angeles, CA 90095-1567 \\ ${ }^{\mathrm{b}}$ Department of Earth and Space Sciences, University of California Los Angeles, \\ CA 90095-1567 \\ ${ }^{\mathrm{c}}$ Laboratoire de Physique de la Matière Condensée, CNRS UMR 6622 and \\ Université de Nice-Sophia Antipolis, 06108 Nice Cedex 2, France
}

\begin{abstract}
In the aftermath of the burst of the "new economy" bubble in 2000, the Federal Reserve aggressively reduced short-term rates yields in less than two years from $6^{1 / 2}$ to $1^{1 / 4} \%$ in an attempt to coax forth a stronger recovery of the US economy. But, there is growing apprehension that this is creating a new bubble in real estate, as strong housing demand is fuelled by historically low mortgage rates. Are we going from Charybdis to Scylla? This question is all the more excruciating at a time when many other indicators suggest a significant deflationary risk. Using economic data, Federal Reserve Chairman A. Greenspan and Governor D.L. Kohn dismissed recently this possibility. Using the theory of critical phenomena resulting from positive feedbacks in markets, we confirm this view point for the US but find that mayhem may be in store for the UK: we unearth the unmistakable signatures (log-periodicity and power law super-exponential acceleration) of a strong unsustainable bubble there, which could burst around the end of the year 2003.
\end{abstract}

Key words: Real estate; Bubble; Econophysics

1 Corresponding author. Department of Earth and Space Sciences and Institute of Geophysics and Planetary Physics, University of California, Los Angeles, CA 90095-1567, USA. Tel: +1-310-825-2863; Fax: +1-310-206-3051. E-mail address: sornette@moho.ess.ucla.edu (D. Sornette)

http://www.ess.ucla.edu/faculty/sornette/

Preprint submitted to Elsevier Science 22 October 2018 


\section{Introduction}

While the US economy has generally been contracting in the last two years, real estate has been growing: house prices have been rising at a rate of about 2 per cent a year faster than income gains. Real consumer outlays and spending on residential construction each rose about 3 percent during 2001. Meanwhile, the gross domestic product (GDP) fell about one-half percent, a drop that would have been far worse without a strong real estate sector. While stock market losses have destroyed maybe as much as \$US 5 trillion in investor wealth since the market's peak, there has been an offsetting effect in the real estate market. Home equity has gained about \$US 1.7 trillion in the same period, according to the chief economist at the biggest US mortgage firm, Fannie Mae. Since, according to the Federal Reserve, home values have double the impact on consumer spending that stock values have via the "richness effect," the housing boom has offset almost two-third of the the stock market losses on the economy.

Such offsets have triggered talks about a real estate bubble in the US. Investment weekly Barron's claimed to spot a "bubble mentality" last April 2002 and analysts are increasingly scrutinizing the possible evidence. A managing director of Pacific Investment Management Co (PIMCO), the largest America bond fund, agrees there is "potential for a bubble in the US residential property market" as a result of the lowest mortgage rates since the 1960s. The statistics released every month continue to confirm that "the housing sector continues to defy all odds," in the words of the chief economist for the National Association of Realtors, David Lereah. Sales of existing housing have been and are continuing to run at a robust if not enthusiastic pace. Total mortgage debt outstanding has risen sharply during the last decade. While the total was about \$US 2.7 trillion in the first quarter of 1990, by the fourth quarter of 1999, it had almost doubled, to \$US 5.2 trillion. As a comparison, the total amount of cumulative borrowing by the Federal Treasury, (the national debt), was about \$US 5.7 trillion in August 2000. American mortgages are on the path of becoming the single largest class of fixed income securities on the planet. Add to these elements that the demand for mortgage borrowing outstrips aggregate domestic saving (which is currently negative and has reached in the last months the lowest level since record keeping began in 1959). This negative saving rate combined with the continuing rapid growth of mortgage borrowing implies that there must be a reduction in non-mortgage lending or an increase in fund flows from abroad or both [1]. This may lead to an increased instability through globalization, resulting from the behavior of international investors [2]. To make things look even worse, the real estate bubble is part of a general huge credit "bubble" that has developed steadily over the last decades, which includes the various US federal money supply, the personal, municipal, corporate debt and federal debts (estimated by some 
to add up to as much as several tens of trillion US dollars), which may not only drag down the recovery of the economy but also lead to vulnerability to exogenous crises.

But is there really a real estate bubble? The science of complexity, which studies the emergence of organization in systems as diverse as the human body (biology), the earth (geology) or the cosmos (astrophysics), suggests novel insights in this troubling question. The science of complexity explains the spontaneous occurrence of coherent large-scale collective behavior, such as well-functioning capitalistic markets but also financial crashes and depressions, from the repeated nonlinear interactions between the constituents of economies. This bottom-up mechanism explains the robustness and strength of modern developed economies as well as their vulnerability to endogenous instabilities. The theory of complex systems thus explains the origin of Adam Smith's invisible hand in society according to which a collection of selfish self-centered individuals coldly maximizing their individual "utility functions" achieve an optimal aggregate social welfare. This theory explains capitalism and free trade. However, it also explains and predicts the occurrence of instabilities and of far-from-optimal equilibria, which are inherent in the bottom-up self-organization [3].

Recent academic research in the field of complex systems suggest that the economy as well as stock markets self-organize under the competing influences of positive and negative feedback mechanisms ([3] and references therein). Positive feedbacks, i.e., self-reinforcement, refer for instance to the fact that, conditioned on the observation that the market has recently moved up (respectively down), this makes it more probable to keep it moving up (respectively down), so that a large cumulative move may ensue. "Positive feedback" is the opposite of "negative feedback", the latter being a concept well-known for instance in population dynamics in the presence of scarce resources. Rational markets and stable economic equilibria derive from the forces of negative feedback. When positive feedback forces dominate, deviations from equilibrium lead to crises. Such instabilities can be seen as intrinsic endogenous progenies of the dynamical organization of the system. Positive feedbacks lead to collective behavior, such as herding in buys during the growth of bubbles and in sells during a crash. This collective behavior does not require the coordination of people to take the same action but results from the convergence of selfish interests together with the impact of interactions between people through the complex network of their acquaintances.

The analysis presented below relies on a general theory of financial crashes and of stock market instabilities developed in a series of works. We refer to Refs. [35] and references therein for all details of our approach. In a nutshell, we are looking for signatures of a faster-than-exponential growth and its decoration by log-periodic oscillations. The faster-than-exponential (super-exponential, 
hyperbolic or power law) growth means that the growth rate itself grows, signaling an unsustainable regime. We add the important ingredient that the log-periodic oscillations have been found to be reliable indicators of endogenous bubbles signaling a coming instability or change of regime [6]. Using these criteria, we find no evidence whatsoever of a bubble in the US real estate market. However, the same analysis applied to the UK real estate market shows that these two signatures of an unsustainable bubble are unambiguously present. Since these signatures have been found to be reliable predictors of past crashes in financial markets, they point to the end of the UK real estate bubble possible around the end of the year 2003, with either a crash or a change of regime in the UK housing market. We should however caution that the crash is only one possible scenarios according to the theory coupling rational expectation bubbles with collective herding behavior [7-9].

Technically, we fit the house price indices to the following versions of the model. In the first version, the logarithm of the price is given by

$$
\ln [p(t)]=A+B\left(t_{c}-t\right)^{m}+C\left(t_{c}-t\right)^{m} \cos \left[\omega \log \left(t_{c}-t\right)-\phi\right]
$$

where $\phi$ is a phase constant, $0<m<1$ quantifies the acceleration of the price, $A=\log \left[p\left(t_{c}\right)\right]$ and $B$ and $C$ are two amplitudes. $B<0$ signals an upward acceleration. This first version (1) amounts to assume that the potential correction or crash at the end of the bubble is proportional to the total price [10]. In contrast, the second version assumes that the potential correction or crash at the end of the bubble is proportional to the bubble part of the total price, that is to the total price minus the fundamental price [10]. This gives the following price evolution:

$$
p(t) \approx A+B\left(t_{c}-t\right)^{m}+C\left(t_{c}-t\right)^{m} \cos \left[\omega \log \left(t_{c}-t\right)-\phi\right] .
$$

Finally, we shall use expression (3) given below, which incorporates higherorder harmonics beyond the first-order log-periodic cosine of formula (1). Indeed, the spectral Lomb analyses reported in section 2.3 suggests the presence of a very strong harmonic at the angular log-frequency $2 \omega$. The possible importance of harmonics was also noticed and used in our recent analysis of many stock markets in the anti-bubble regime that started worldwide during the summer of 2000 [5,11]. This followed the analyses of log-periodicity in hydrodynamic turbulence data $[12,13]$ which have demonstrated the important role of higher harmonics in the detection of log-periodicity. In view of the parsimony and quality of the fit with expression (3), we shall use it for our test of robustness of the estimation of the critical time $t_{c}$. 


\section{Speculative bubble in UK real estate}

\subsection{Data sets}

Since 1984, the Halifax house price index has been used extensively by government departments, the media and businesses as an authoritative indicator of house price movements in the United Kingdom. This index is based on the largest sample of housing data and provides the longest unbroken series of any similar UK index. We use this monthly house price index data that are retrieved from the web site of HBOS $\mathrm{plc}^{2}$. Six data sets are analyzed as listed in Table 1. Each data set is a time series, which starts in December 1992 and ends in April 2003.

\subsection{Log-periodic pattern}

In order to identify the speculative real estate bubble in the UK market, we fit Eq. (1) to the six data sets described in Table 1, following the fitting procedure described in detail in [5].

Figure 1 shows the best fits of Eq. (1) to the UK house price index for the six data sets. For the sake of presentation, we shift the curves downward by multiples of 0.15 : the curve at the top corresponds to the set 1 , the curve immediately below translated by 0.15 vertically corresponds to set 2 , the following curve translated again by 0.15 vertically corresponds to set 3 , and so on. The vertical lines indicate the values of the critical time $t_{c}$ obtained from the fit of Eq. (1) to each of the six data sets. Recall that $t_{c}$ is the expected time for the end of the bubble. It corresponds to the most probable time for a change of regime or a crash to occur $[7,8]$. However, a crash could still occur before, albeit with small probability.

The log-periodic power-law signatures are clearly visible to the naked eye. The values of the fitted parameters are listed in Table 2. One observes that the predicted $t_{c}$ are consistent for the six data sets and the values of the angular $\log$-frequencies $\omega$ indicate the existence of a fundamental angular log-frequency around $\omega \approx 5.8$.

Figure 2 presents the best fits of Eq. (2) to the UK house price index (and not their logarithm as in Figure 1) for the six data sets described in Table 1. We also translate the curves as in Figure 1 in the same way with a vertical shift of 35 downward for each successive curve. Again, the vertical lines indicate

$\overline{2}$ http://www.hbosplc.com/view/housepriceindex/housepriceindex.asp. 
the positions of the critical times $t_{c}$ for each data set. The log-periodic powerlaw signatures are again clearly visible. The values of the fitted parameters are given in Table 3 . While the predicted critical times $t_{c}$ are still quite consistent, they exhibit a significantly larger scatter than when using Eq. (1), suggesting that the bubble is better described by a pure speculative component given by Eq. (1). This conclusion is strengthened by the fact that the fits with Eq. (2) give negative exponents $m$, corresponding to a divergence of the price (in contrast with the divergence of the change of trend of the price described by the fits with Eq. (1)). Eq. (1) seems thus more consistent with a reasonable price trajectory, exhibiting super-exponential acceleration, but culminating at a finite value with infinite unsustainable slope. This again suggests a very strong component of the bubble component to the price, making the fundamental component undetectable in this analysis.

In contrast, the found angular log-frequencies $\omega$ are very robust with respect to the choice of Eq. (1) versus Eq. (2) and retrieve a fundamental value $\omega \approx 6.8$. This confirms to us the existence of a very clear speculative signal in these price time series. The consistency of the results across the six different ways of calculating the House price indices also suggests that we are deciphering here a very strong speculative component of the dynamics of house prices, which remain robust whatever the specific nature of the house and the way they are estimated.

\subsection{Significance of the log-periodic patterns}

To assess the significance level of the extracted log-periodic pattern, we complement the parametric analysis of Eq. (1) with non-parametric analyses.

For our first test, we use the first two terms $A+B\left(t_{c}-t\right)^{m}$ of the righthand-side of equation (1) to detrend $\ln [p(t)]$, that is, we construct the residual $\left\{\ln [p(t)]-A-B\left(t_{c}-t\right)^{m}\right\} /\left(t_{c}-t\right)^{m}$, in the manner introduced in Ref. [7]. A perfect sinusoidal log-periodicity would correspond to this residual being a perfect cosine $\cos \left[\omega \log \left(t_{c}-t\right)+\phi\right]$. In order to quantify the log-periodic oscillatory components of the six time series, we calculate the Lomb periodograms (which are similar to a Fourier transform for unevenly spaced data) of the corresponding residues for the six data sets.

Figure 3 gives the Lomb periodograms of the detrended residuals of the six house price indices and their Lomb average as the thickest line. All periodograms present two significant peaks around $\omega_{1}=5.9$ and a value $\omega_{2}=13.0$ close to its harmonics $2 \omega_{1}$.

Our second test consists in performing non-parametrically the $(H, q)$-analysis of the logarithm of the price as described in Refs. [14,5]. In a nutshell, the 
$(H, q)$-analysis performs a kind of fractal derivative which is particularly sensitive to the presence of log-periodicity. The index $q$ refers to the discrete scaling ratio used in the definition of the fractal $q$-derivative. The index $H$ is the exponent used to rescale the $q$-derivative. Scanning $q$ and $H$ provides an important test of the robustness of the log-periodic signal. A Lomb periodogram analysis of the $(H, q)$-derivative allows one to detect the presence of significant log-periodicity. Figure 4 shows the angular log-frequency $\omega$ of the most significant Lomb peak in each Lomb periodogram for each of the scanned $(H, q)$ pairs of $(H, q)$-derivative $[14,15]$ of the logarithm of the price of one of the house price index. The middle and high plateaus show respectively the fundamental log-frequency and its harmonic. The lower level corresponds to the artificial log-frequency corresponding to the most probable noise of a power law signal demonstrated in Ref. [16].

The analysis of the power law residuals and the $(H, q)$-analysis confirm the presence of very significant log-periodicity in the UK house price indices over the last decade.

\subsection{Parametric fit taking into account the second log-periodic harmonics}

Figures 3 and 4 have demonstrated the strong amplitude of a second logperiodic harmonic. Similarly to our previous modelling of the stock markets $[5,11,17]$, we extend (1) into

$$
\ln [p(t)]=A+B \tau^{m}+C \operatorname{Re}\left(\sum_{n=1}^{N} n^{-m-0.5} \mathrm{e}^{i \psi_{n}} \tau^{-s_{n}}\right),
$$

where $\tau=\left(t_{c}-t\right) / T$ ( $T$ determines the time units), $A, B$ and $C$ are three parameters, $N$ is the number of log-periodic harmonics kept in the description, and

$$
s_{n}=-m+i \frac{2 \pi}{\ln \gamma} n .
$$

The operator $\operatorname{Re}(x)$ takes the real part of $x$. In the second term of the righthand-side of (3), we have used $s_{n=0}=-m$ as seen from (4). Note that the case $N=1$ recovers Eq. (1). The function (3) is called a Weierstrass-type function [18].

Since the choice $\psi_{n}=0$ plays an essential role in the modelling of financial time series in the bubble and/or anti-bubble regimes as shown in [17,18], we use Eq. (3) with $\psi_{n}=0$ and follow the procedure described in [17] to fit the six data sets. This choice, which corresponds to have the same phases for all 
harmonics, has also the advantage of keeping constant the number of degrees of freedom such that the more complex formula (3) has the same number of free parameters as the simplest log-periodic equation (1). By this model (3), we thus gain in relevance while keeping the same parsimony.

Figure 5 shows the best fits of Eq. (3) with $\psi_{n}=0$ and $N=2$ to the six UK house price indices described in Table 1. The log-periodic signatures are again clearly visible. The values of the fitted parameters are listed in Table 4. Compared with Table 2, one can see that the present fits give much better goodness-of-fit with smaller r.m.s. errors. Since they have the same number of free parameters, formula (3) should be prefered as a better model than equation (1). The angular log-frequency is found to be very stable and close to the fundamental value around 6 identified in previous fits. This increased stability stems from the fact that expression (3) automatically accounts for higher harmonics up to order $N$. This result strengthens the existence and evidence for strong log-periodicity harmonics. The exponents $m$ and critical time $t_{c}$ are consistent across the six time series. Taking into account higherorder harmonics $N>2$ does not improve the fits significantly due to the coarse monthly sampling rate and the marginal importance of the third-order and four-order log-periodic harmonics shown in Figure 3.

\subsection{Test of the robustness of the critical time $t_{c}$}

The determination of $t_{c}$ is particularly important as it gives the estimated termination time of the speculative bubble as well as the most probable time for a crash, if any. Beyond $t_{c}$, the speculative bubble has to transform into another regime because its path before and up to $t_{c}$ has become unsustainable. We stress that several models of stochastic speculative bubbles indicate that a bubble does not end necessarily with a crash as there is a finite probability for a bubble to end by a transition to another regime such as slow deflation $[7,8,19] . t_{c}$ is thus not the time of the crash, it is both the end of the bubble and the time at which the crash is most probable, if it ever occurs. Nevertheless, there is an obvious interest in estimating when the speculative trend of the real estate bubble in the UK will end up.

As an attempt to address this question, we investigate the robustness of the estimated critical time $t_{c}$ obtained from the previous fits. For this, we perform again fits with Eq. (3) of the six time series from December 1992 to a time $t_{\text {last }}$ and vary $t_{\text {last }}$ to see if $t_{c}$ has remained approximately constant in the past. For each $t_{\text {last }}$, we report in Figure 6 the value $t_{c}$ of the best fit with Eq. (3) for each of the 6 time series. Except for time series 2 (all, non seasonally adjusted), all other 5 seasonally adjusted time series are grossly consistent with a critical time $t_{c}$ that could fall anywhere from the present to the end of the year 2003 . 
Figure 6 conveys two pieces of information. First, the description of the data with the log-periodic formula (3) with two log-frequencies is found approximately stable as a function of time. Second, the precision with which one can pinpoint the specific end of the presently unfolding speculative bubble in the UK house prices is limited. In this vein, the behavior of the critical time $t_{c}$ predicted with time series 2 (all, non seasonally adjusted) is instructive: after a plateau, $t_{c}$ is found to grow systematically with $t_{\text {last }}$. This is a property which has been found in past investigations (see Chapter 9 of [3]) to be due to the fact that the real end of the bubble is beyond the time horizon over which the log-periodic fits provide reliable predictors. When using only the first log-periodic harmonics with expression (1), a monotonous increase of the predicted $t_{c}$ 's with $t_{\text {last }}$ is found for all 6 time series, which is similar to that found with time series 2 using Eq. (3). Together with the results shown in Figure 6, this confirms the stronger relevance of Eq. (3): the addition of a second log-periodic harmonics seems to extend the time-horizon over which forecasts are stable. This also shows that the true end of the bubble is beyond the time horizon of formula (1).

Finally, we should note the significantly smaller variance of the predicted $t_{c}$ using for $t_{\text {last }}$ the last three available data.

\section{Exponential growth in the USA real estate market}

Figure 7 shows the deflated quarterly average sales prices $p(t)$ of new houses sold in all the states in the USA and by regions (northeast, midwest, south and west) in the last decade as a function of time $t$. The data taken from the U.S. Census Bureau ${ }^{3}$ are deflated by the consumer price index of the USA available at the Bureau of Labor Statistics ${ }^{4}$. The linear dependence of $\ln [p(t)]$ against $t$ in this semi-log plot qualifies clearly an exponential growth

$$
p(t)=p(0) \mathrm{e}^{r t}
$$

with a constant growth rate $r$. The yearly growth rates found by linearly regressing $\ln p(t)$ against time are $2.3 \%$ (all states), $2.5 \%$ (northeast), $1.6 \%$ (midwest), $1.9 \%$ (south) and 3.5\% (west). Exactly the same behavior is found (not shown) on non-deflated prices; simply, the growth rates are different $(4.7 \%$ (all states), 4.1\% (northeast), 5.3\% (midwest), 3.9\% (south) and 3.9\% (west)).

Using expression (1) to fit these same data sets since December 1992 to December 2002, we find absolutely no significant log-periodicity and no power-

3 http://www.census.gov/const/www/newressalesindex.html.

4 http://www.bls.gov/data/ 
law criticality in the investigated price trajectory. According to the proposal $[19,3,6]$ that speculative bubbles are associated with super-exponential growth with significant log-periodicity, the recent house price time series in the US do not qualify as bubbles.

The USA real estate market has been rather segmented because of its size which is not comparable to the UK market where London plays a leading role. It would thus be more rational to compare the UK with California for instance, as the two markets have approximately the same geographical size and level of integration, with Los Angeles and San Francisco leading the Californian market. In Fig. 8, we plot the logarithm of house price indices for California, Los Angeles and San Francisco, respectively. The house price indices are also deflated by the USA consumer price index for inflation. Note that the data for Los Angeles and San Francisco are normalized to 100 in the first quarter of 1995, while that for California is normalized to 100 in the first quarter of 1980. The difference in normalization dates has no impact on appreciation rates obtained for each index. The two vertical lines delimit a super-exponential growth, which ended at the end of 1989, and which paralleled the Japan real estate bubble in the 1980's and its burst at the end of 1989. The real estate bubble ending in 1990 has been well-documented and it is interesting to find its super-exponential signature so-clearly in this analysis. For the present, while there is undoubtedly a strong growth rate, there is no evidence of a superexponential growth in the latest six years. Similar conclusions hold for the un-deflated indices.

\section{Conclusion}

Testifying before the Senate Committee on Banking, Housing and Urban Affairs in July 2002, Federal Reserve Board Chairman Alan Greenspan told lawmakers that rising home prices in the USA are a by-product of "low mortgage rates, immigration, and shortages of buildable land in some areas." As a result, homeowners have more equity they can use to pay off high-cost consumer debt and for other purposes. This leads to a beneficial effect on the US economy rather than suggesting the possibility of a real estate crash.

Based on the science of complexity, our analysis provides a confirmation of this conclusion derived from more standard economic analysis.

The situation is the opposite for the UK market. Our same analysis applied to the UK real estate market shows two unambiguous signatures of an unsustainable bubble, which started years even before the end of the stock market bubble in 2000. These signatures have been found to be reliable predictors of past crashes in financial markets. The analysis points to the end of the 
bubble around the end of the year 2003, with either a crash or a change of direction in the UK housing market. While there are very strong correlations between stock markets in developed countries at present [11], no such correlation has yet materialized in real estate markets. Investors should however remain watchful for indications of a possible contagions to the US in the longer term.

To summarize, what we have learned in this paper is that (i) the USA real estate market is in a state compatible with "rational expectation" regime; (ii) the UK real-estate market exhibits an ultimately unsustainable speculative bubble; (iii) The UK house prices will continue going up during the year 2003; and (iv) The Weierstrass-type function (3) outperforms the simple log-periodic power law formula.

\section{Acknowledgments}

We acknowledge stimulating discussions with D. Darcet and B. Roehner and a referee for suggestions and thank T. Gilbert for help. This work was supported in part by the James S. Mc Donnell Foundation 21st century scientist award/studying complex system.

\section{References}

[1] R. Roll, Benefits to homeowners from mortgage portfolios retained by Fannie Mae and Freddie Mac, working paper (2002).

[2] J.E. Stiglitz, Globalization and its discontents, 1st ed. (New York: W.W. Norton, 2002).

[3] D. Sornette, Why Stock Markets Crash: Critical Events in Complex Financial Systems (Princeton University Press, Princeton, NJ, 2003).

[4] D. Sornette and A. Johansen, Significance of log-periodic precursors to financial crashes, Quantitative Finance 1 (2001) 452-471.

[5] D. Sornette and W.-X. Zhou, The US 2000-2002 Market Descent: How Much Longer and Deeper? Quantitative Finance 2 (2002) 468-481.

[6] A. Johansen and D. Sornette, Endogenous versus Exogenous Crashes in Financial Markets, preprint at cond-mat/0210509.

[7] A. Johansen, O. Ledoit and D. Sornette, Crashes as critical points, International Journal of Theoretical and Applied Finance 3 (2000) 219-255.

[8] A. Johansen, D. Sornette and O. Ledoit, Predicting Financial Crashes using discrete scale invariance, Journal of Risk 1 (1999) 5-32. 
[9] D. Sornette and W.-X. Zhou, Predictability of Large Future Changes in Complex Systems, preprint at cond-mat/0304601

[10] A. Johansen and D. Sornette, Bubbles and anti-bubbles in Latin-American, Asian and Western stock markets: An empirical study, International Journal of Theoretical and Applied Finance 4 (2001) 853-920.

[11] W.-X. Zhou and D. Sornette, Evidence of a Worldwide Stock Market LogPeriodic Anti-Bubble Since mid-2000, preprint at cond-mat/0212010.

[12] W.-X. Zhou and D. Sornette, Evidence of Intermittent Cascades from Discrete Hierarchical Dissipation in Turbulence, Physica D 165, 94-125 (2002).

[13] W.-X. Zhou, D. Sornette and V. Pisarenko, New Evidence of Discrete Scale Invariance in the Energy Dissipation of Three-Dimensional Turbulence: Correlation Approach and Direct Spectral Detection, Int. J. Modern Phys. C 14 (2003) 459-470.

[14] W.-X. Zhou and D. Sornette, Generalized q-Analysis of Log-Periodicity: Applications to Critical Ruptures, Phys. Rev. E (2002) 046111.

[15] W.-X. Zhou and D. Sornette, Non-Parametric Analyses of Log-Periodic Precursors to Financial Crashes, in press in Int. J. Mod. Phys. C, preprint at cond-mat/0205531.

[16] Y. Huang, A. Johansen, M. W. Lee, H. Saleur and D. Sornette, Artifactual Log-Periodicity in Finite-Size Data: Relevance for Earthquake Aftershocks, J. Geophys. Res. 105 (2000) 25451-25471.

[17] W.-X. Zhou and D. Sornette, Renormalization Group Analysis of the 20002002 anti-bubble in the US S\&P 500 index: Explanation of the hierarchy of 5 crashes and Prediction, preprint at physics/0301023.

[18] S. Gluzman and D. Sornette, Log-periodic route to fractal functions, Phys. Rev. E 65 (2002) 036142.

[19] D. Sornette and J.V. Andersen, A Nonlinear Super-Exponential Rational Model of Speculative Financial Bubbles, Int. J. Mod. Phys. C 13 (2002) 171188. 
Table 1

The UK real estate price indices used in this paper.

\begin{tabular}{cll}
\hline \hline No. & Name & Description \\
\hline 1 & All(SA) & All Houses (All Buyers), Seasonally Adjusted \\
2 & All(NSA) & All Houses (All Buyers), Non Seasonally Adjusted \\
3 & New & New Houses (All Buyers), Non Seasonally Adjusted \\
4 & Existing & Existing Houses (All Buyers), Non Seasonally Adjusted \\
5 & FOO & Former Owner Occupiers (All Houses), Non Seasonally Adjusted \\
6 & FTB & First Time Buyers (All Houses), Non Seasonally Adjusted \\
\hline \hline
\end{tabular}

Table 2

Parameters of the fits of the log-periodic function (1) to the logarithm of the real estate price indices in the United Kingdom.

\begin{tabular}{ccccccccc}
\hline \hline No. & $t_{c}$ & $m$ & $\omega$ & $\phi$ & $A$ & $B$ & $C$ & $\chi$ \\
\hline 1 & $2003 / 12 / 10$ & 0.01 & 5.7 & 3.77 & 35.89 & -28.20 & 0.028 & 0.021 \\
2 & $2003 / 12 / 01$ & 0.01 & 5.6 & 5.84 & 35.63 & -27.96 & -0.027 & 0.018 \\
3 & $2003 / 12 / 21$ & 0.01 & 5.8 & 1.18 & 37.11 & -29.32 & -0.028 & 0.023 \\
4 & $2003 / 11 / 28$ & 0.01 & 5.7 & 0.28 & 33.09 & -25.66 & -0.032 & 0.029 \\
5 & $2003 / 12 / 22$ & 0.01 & 5.8 & 4.30 & 37.41 & -29.60 & 0.029 & 0.021 \\
6 & $2003 / 12 / 27$ & 0.01 & 6.1 & 0.68 & 34.72 & -27.10 & 0.025 & 0.020 \\
\hline \hline
\end{tabular}


Table 3

Parameters of the fits of the log-periodic function (2) to the real estate price indexes in the United Kingdom.

\begin{tabular}{ccccccccc}
\hline \hline No. & $t_{c}$ & $m$ & $\omega$ & $\phi$ & $A$ & $B$ & $C$ & $\chi$ \\
\hline 1 & $2004 / 06 / 12$ & -0.51 & 6.8 & 3.10 & 81.24 & 7953.58 & -309.910 & 5.207 \\
2 & $2004 / 08 / 13$ & -0.59 & 6.9 & 1.36 & 98.74 & 13306.96 & 545.197 & 4.351 \\
3 & $2004 / 07 / 29$ & -0.56 & 7.0 & 2.15 & 86.32 & 11225.99 & 445.223 & 5.774 \\
4 & $2004 / 02 / 21$ & -0.34 & 6.4 & 5.33 & 29.78 & 2573.31 & -95.704 & 7.531 \\
5 & $2004 / 05 / 18$ & -0.47 & 6.8 & 5.83 & 59.07 & 6274.64 & 232.723 & 5.713 \\
6 & $2004 / 05 / 02$ & -0.44 & 6.9 & 1.00 & 70.50 & 5021.50 & 172.436 & 5.038 \\
\hline \hline
\end{tabular}

Table 4

Parameters of the fits of the log-periodic function (3) to the logarithm of the real estate price indices in the United Kingdom.

\begin{tabular}{cccccccc}
\hline \hline No. & $t_{c}$ & $m$ & $\omega$ & $A$ & $B$ & $C$ & $\chi$ \\
\hline 1 & $2004 / 01 / 08$ & 0.03 & 6.1 & 17.22 & -9.56 & 0.023 & 0.016 \\
2 & $2004 / 01 / 19$ & 0.01 & 6.1 & 37.56 & -29.75 & 0.025 & 0.013 \\
3 & $2004 / 01 / 13$ & 0.03 & 6.1 & 14.97 & -7.28 & 0.022 & 0.018 \\
4 & $2003 / 12 / 20$ & 0.03 & 6.1 & 16.91 & -9.51 & 0.025 & 0.026 \\
5 & $2004 / 01 / 20$ & 0.03 & 6.1 & 17.51 & -9.75 & 0.024 & 0.016 \\
6 & $2003 / 11 / 25$ & 0.07 & 6.1 & 9.49 & -2.29 & 0.015 & 0.016 \\
\hline \hline
\end{tabular}




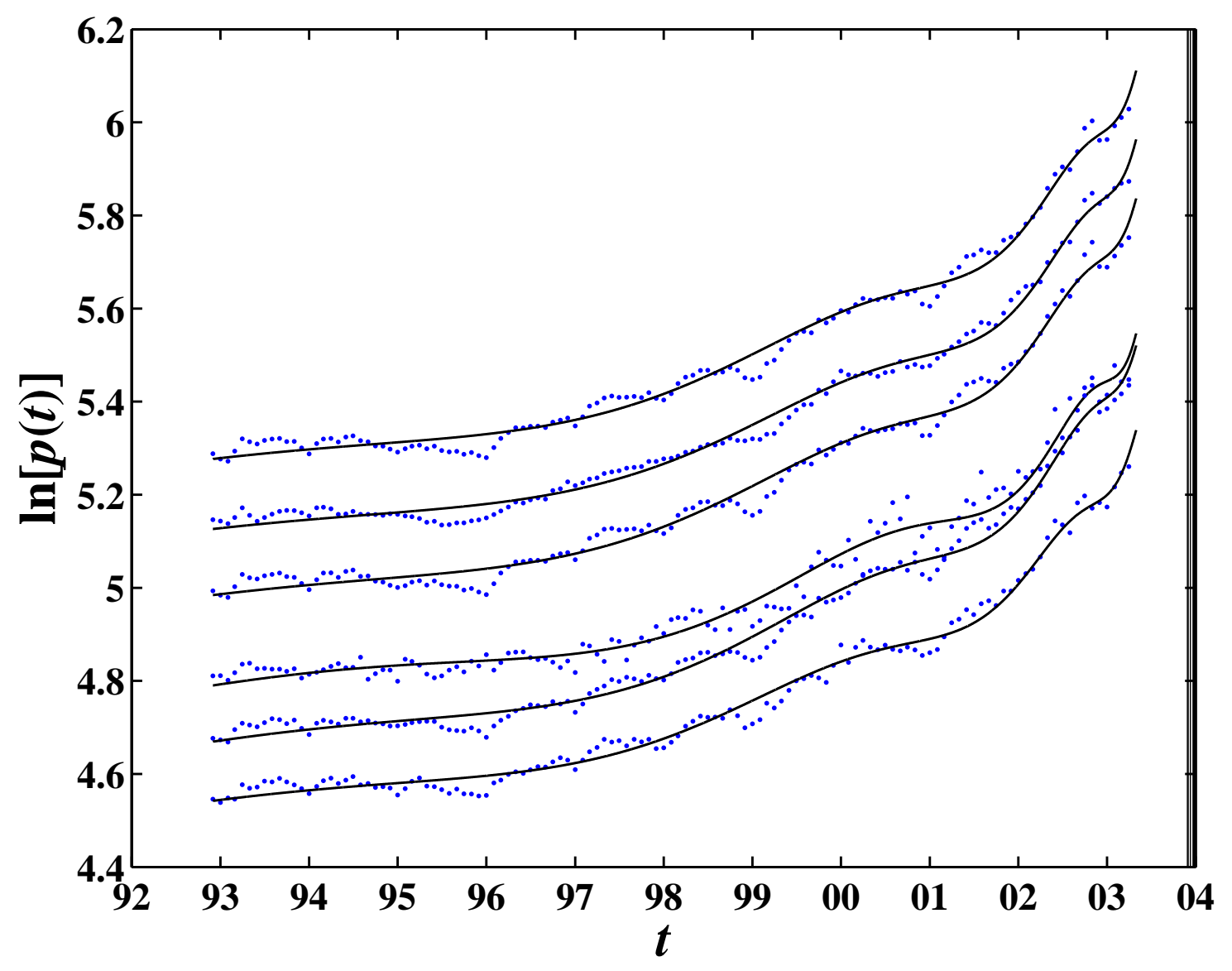

Fig. 1. Best fits of Eq. (1) to the logarithm of the six UK house price indices described in Table 1 from December 1992 to April 2003. The values of the fitted parameters are listed in Table 2 . The curves have been shifted vertically downward by 0.15 incrementally from the first to the sixth index. 


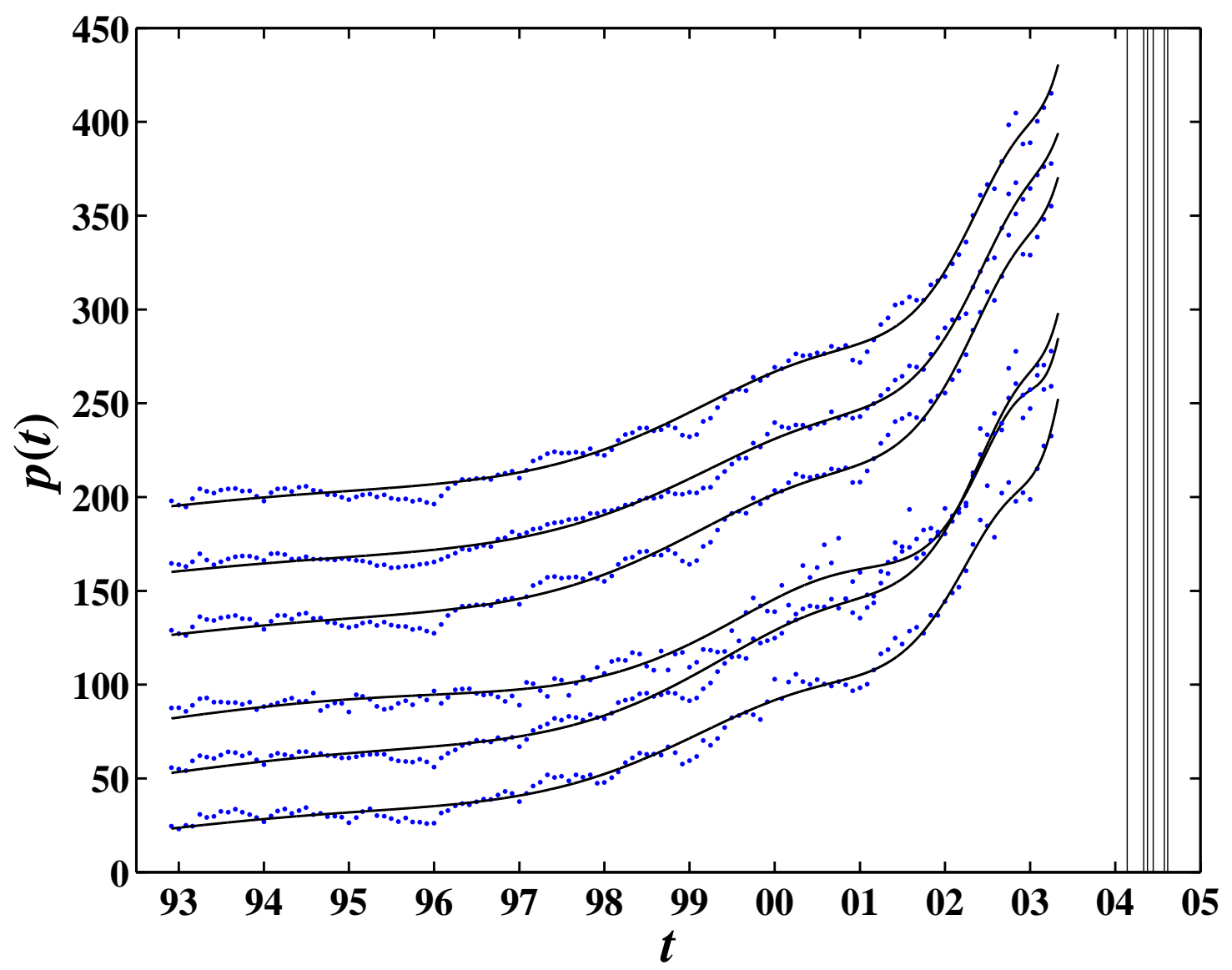

Fig. 2. Best fits of Eq. (2) to the six UK house price indices described in Table 1 from December 1992 to April 2003. The values of fitted parameters are listed in Table 3. The curves have been shifted vertically downward by 35 incrementally from the first to the sixth index. 


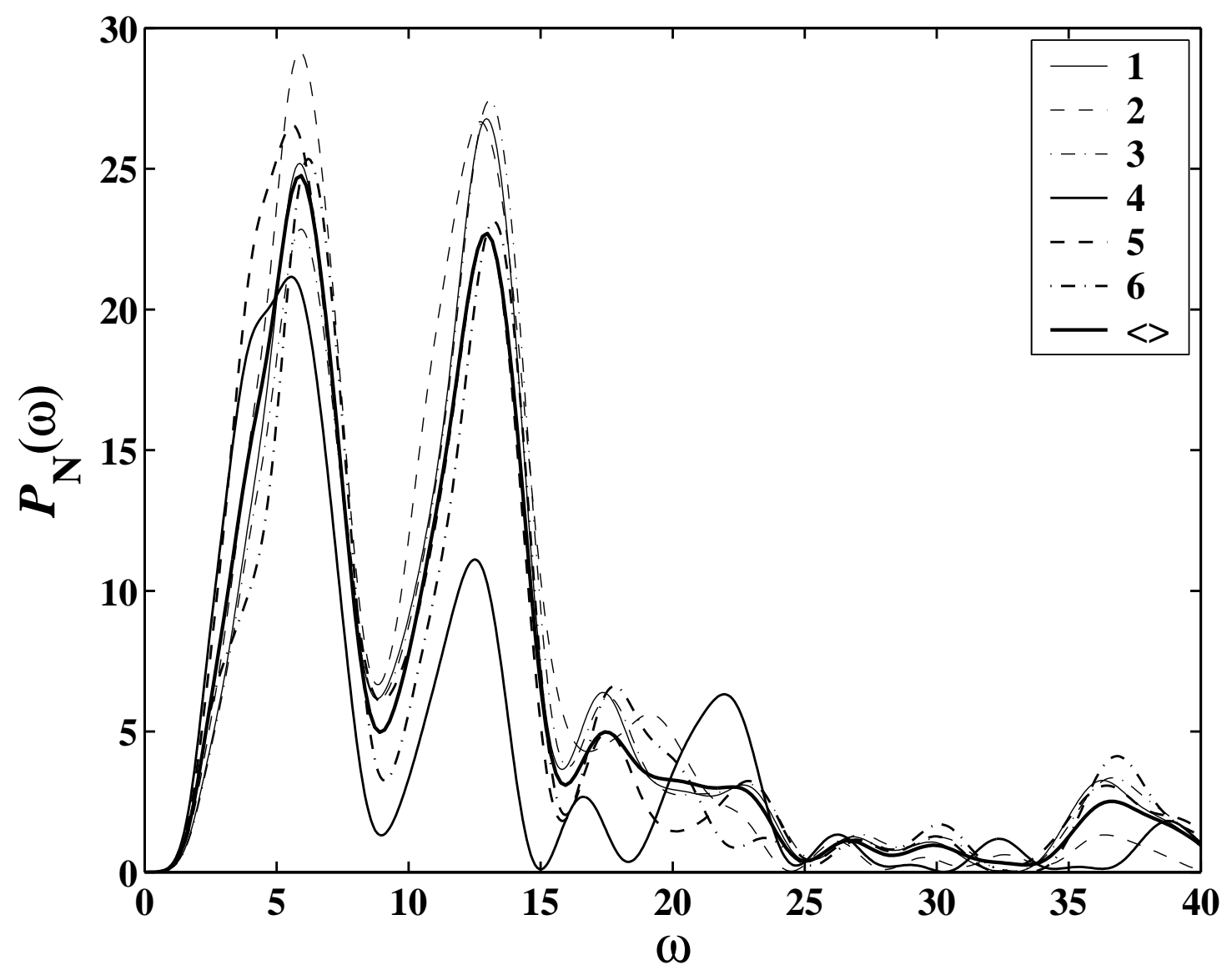

Fig. 3. Lomb periodograms of the detrended residuals of the six house price indexes and their Lomb average as the thickest line (shown in the legend as "<>"). All the periodograms present two significant peaks around $\omega=5.9$ and its harmonic $\omega=13.0$. 


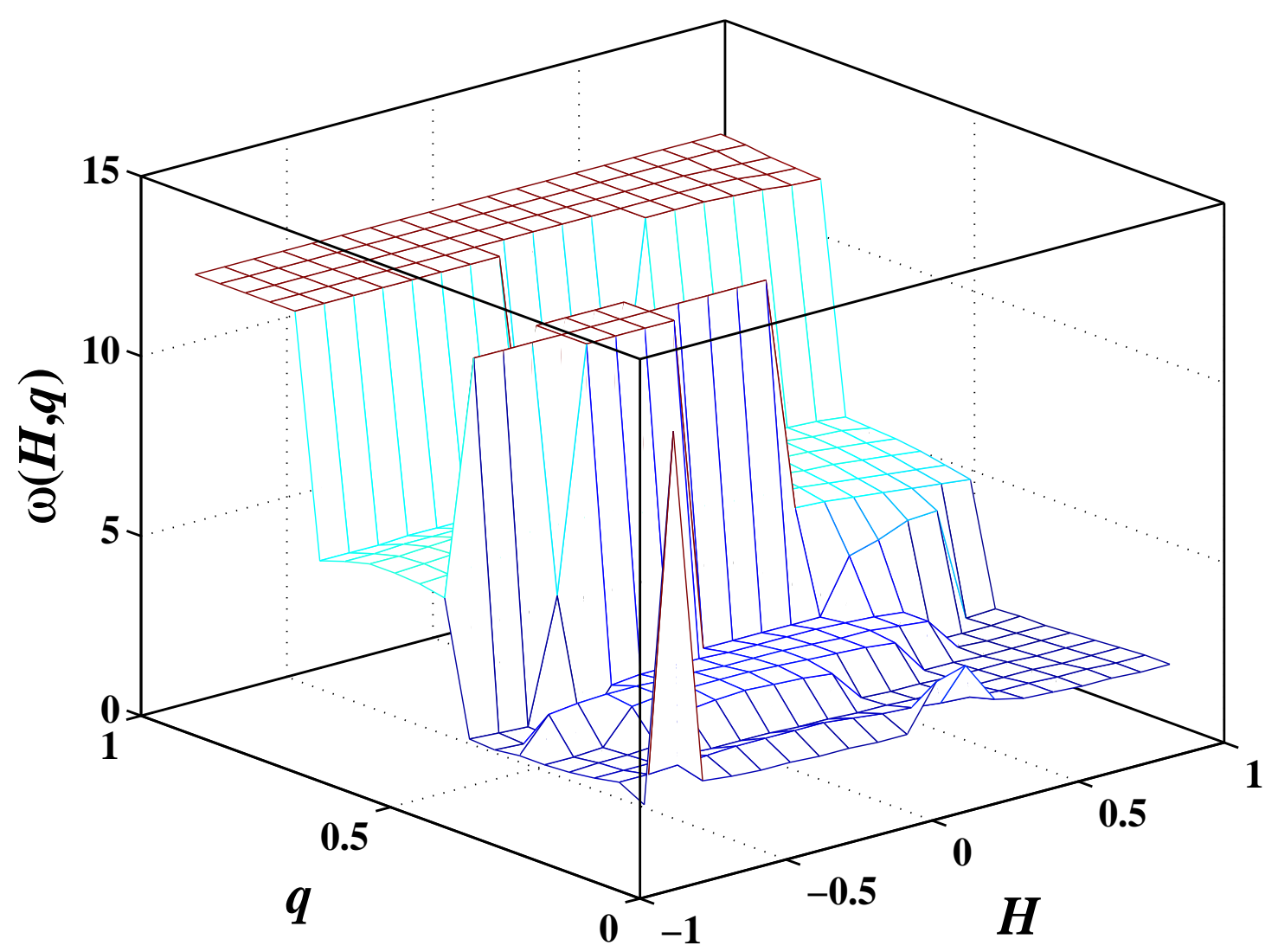

Fig. 4. Angular log-frequency $\omega$ of the most significant Lomb peak in each lomb periodogram in the $(H, q)$-analysis of the logarithm of the price of the seasonally adjusted first time buyers of all houses (FTB). See text for more explanation. The middle and high plateaus show respectively the fundamental log-frequency and its harmonic. The lowest background corresponds to the artificial most probable log-frequency resulting from the most probable noise decorating a power law. 


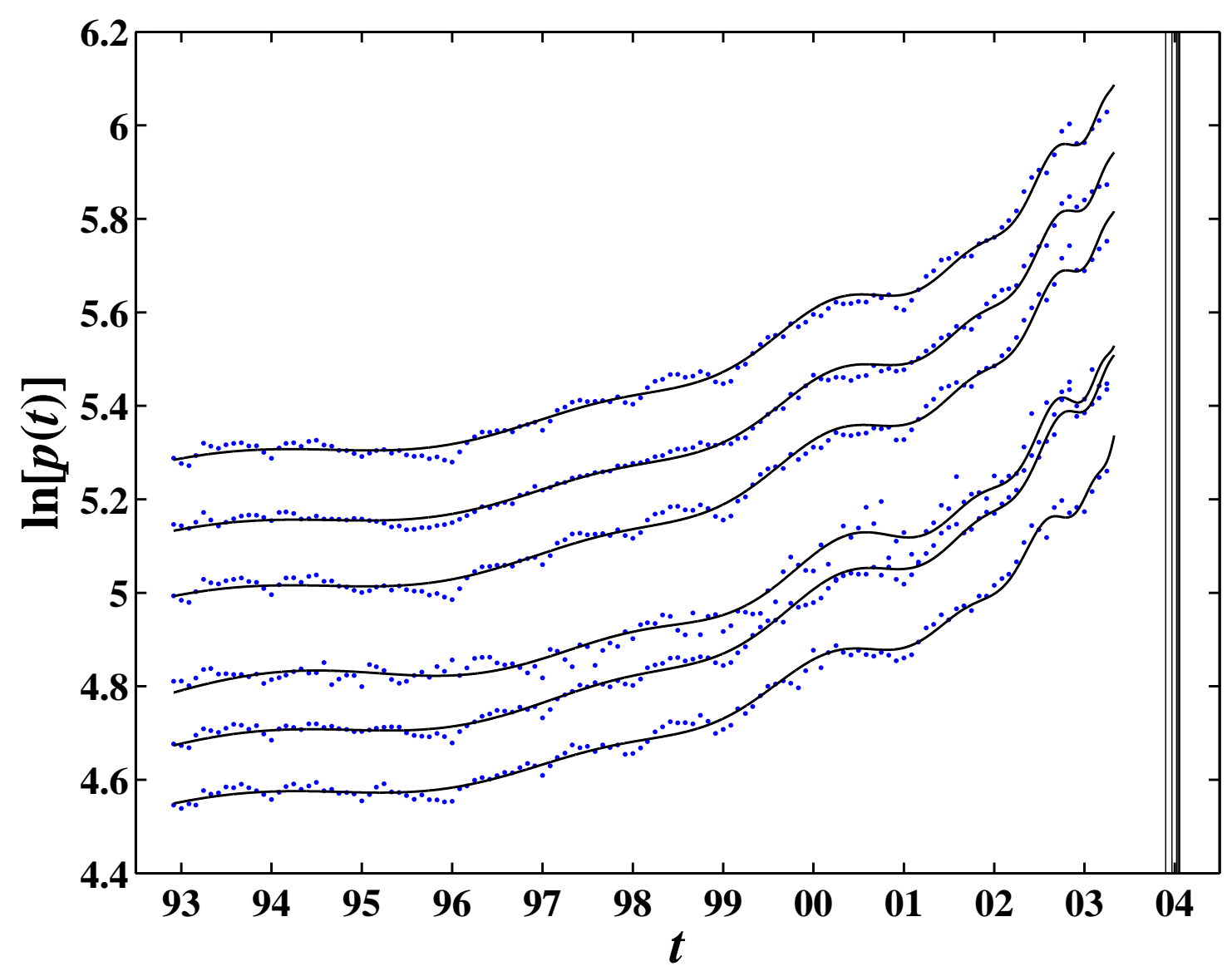

Fig. 5. Best fits of Eq. (3) with $\phi_{n}=0$ and $N=2$ to the logarithm of the six UK house price indices described in Table 1 from December 1992 to April 2003. The values of fit parameters are listed in Table 4 . The curves have been shifted vertically downward by 0.15 incrementally from the first to the sixth index. 


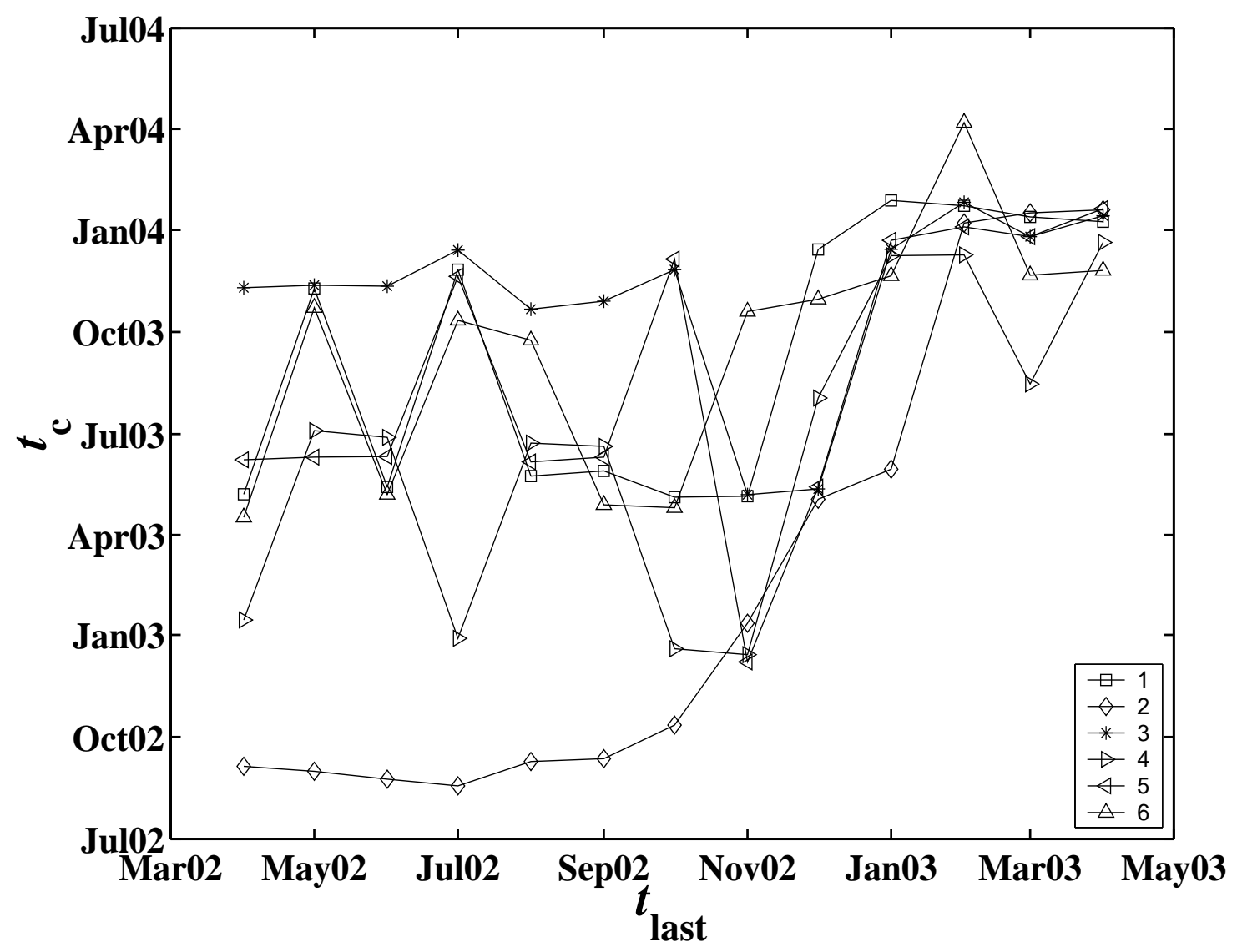

Fig. 6. Test of robustness of the estimated critical time $t_{c}$ obtained by varying the last point $t_{\text {last }}$ of the time series up to which the fits using the formula (3) with $N=2$ and $\psi_{n}=0$ are performed. See text for details of the predition procedure. 


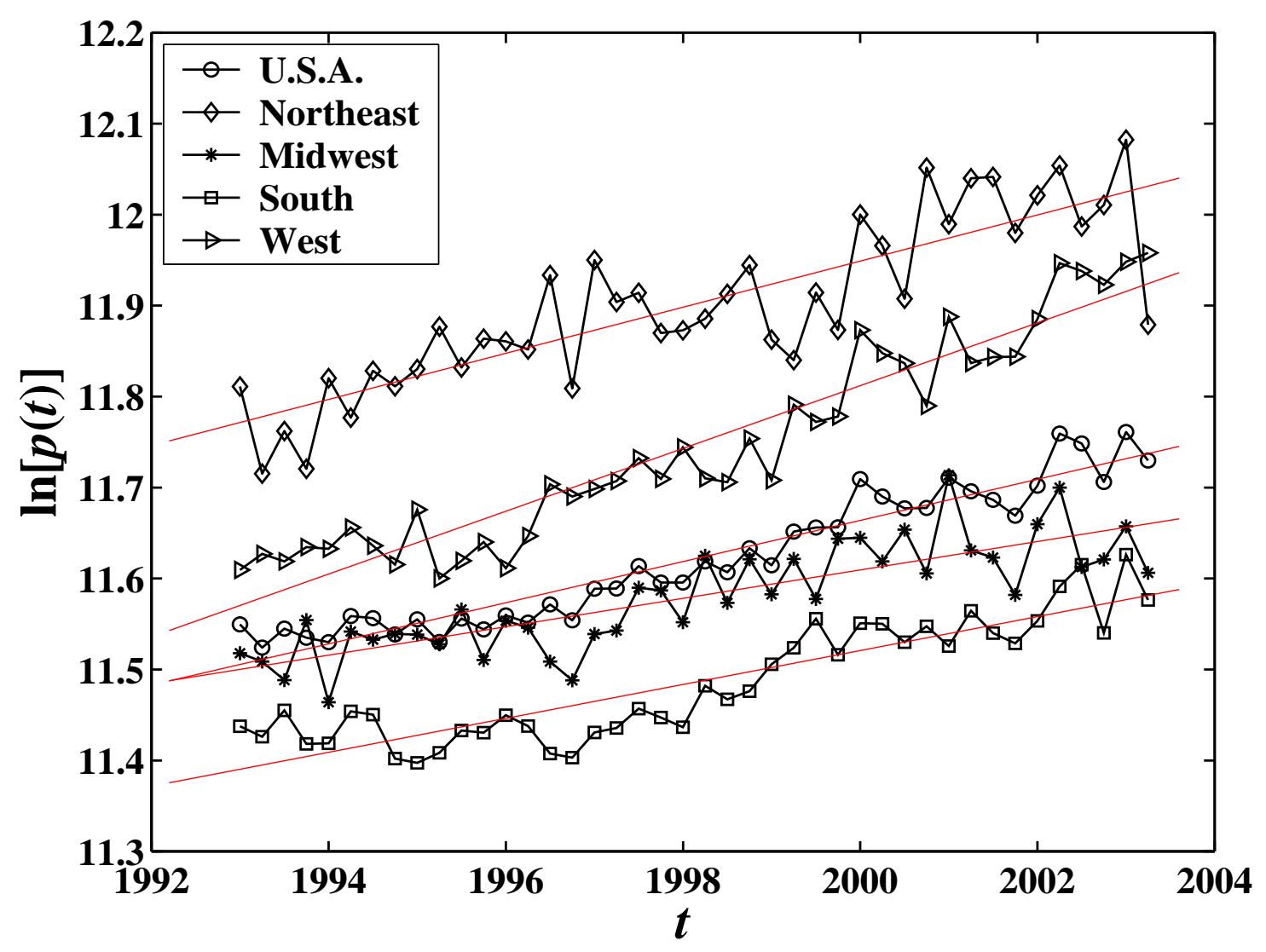

Fig. 7. Deflated quarterly average sale prices $p(t)$ of new houses sold in all the states in USA and by regions (northeast, midwest, south and west) in the last decade as a function of time $t$ in a semi-log representation. The linear dependence of $\ln [p(t)]$ against $t$ implies a stable exponential growth. The yearly growth rates are $2.3 \%$ (all states), $2.5 \%$ (northeast), 1.6\% (midwest), 1.9\% (south) and 3.5\% (west). 


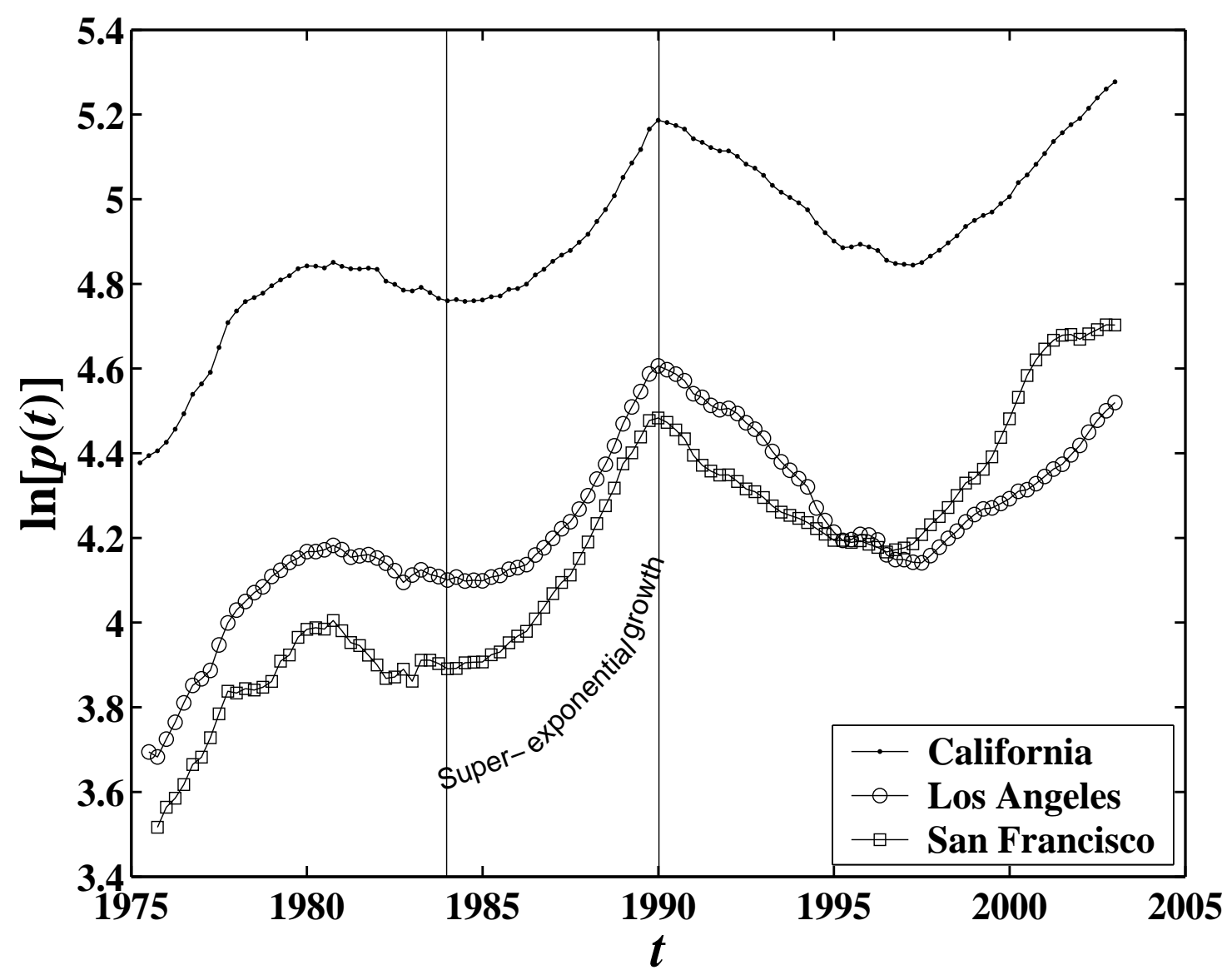

Fig. 8. The logarithm of the deflated house price indexes for California, Los Angeles and San Francisco, respectively. The two vertical lines delimit the super-exponential growth regime which is characteristic of the real estate bubble that ended in 1990 . 